\title{
Automatic analysis of Pole Mounted Auto-Recloser data for fault diagnosis and prognosis
}

\author{
X. Wang \\ Dept. EEE, University of \\ Strathclyde \\ xiaoyu.wang@strath.ac.uk
}

\author{
S. M. Strachan \\ Dept. EEE, University of \\ Strathclyde \\ scott.strachan@strath.ac,uk
}

\author{
S. D. J. McArthur \\ Dept. EEE, University of \\ Strathclyde \\ s.mcarthur@strath.ac.uk
}

\author{
J. D. Kirkwood \\ SP Energy Networks \\ john.kirkwood \\ @SPPowerSystems.com
}

\begin{abstract}
Fault diagnosis is a key part of a control and protection engineer's role to ensure the effective and stable performance of electrical power networks. One challenge is to support the analysis and application of expert judgement to the, often, large data sets generated. To assist engineers with this task and improve network reliability, this research focuses on analysing previous fault activity in order to obtain an earlywarning report to assist fault diagnosis and fault prognosis.
\end{abstract}

This paper details the design of an integrated system with a fault diagnosis algorithm utilising available Supervisory Control And Data Acquisition (SCADA) alarm data and $11 \mathrm{kV}$ distribution network data captured from Pole Mounted AutoReclosers (PMARs) (provided by a leading UK network operator). The developed system will be capable of diagnosing the nature of a circuit's previous fault activity, underlying circuit activity and evolving fault activity and the risk of future fault activity. This will provide prognostic decision support for network operators and maintenance staff.

Keywords- Fault diagnosis; Fault activity; SCADA alarm data; Distribution network data; Decision support; Distribution automation

\section{INTRODUCTION}

Distribution networks are characterised by the large geographic areas they cover, their complex topologies and vast numbers of circuit components. Therefore, it is inevitable that during the network lifetime it will experience many different faults which, given the size of these networks, can be difficult to manage and respond to in a timely manner and within regulatory requirements. When a fault occurs, restoration strategies [1] should locate faults and restore customer supplies as quickly and efficiently as possible (either manually or automatically). This operation requires the location and diagnosis of the fault [2].

During the last few decades, with the technological development of automatic restoration and reconfiguration for quick and efficient customer supplies, fault diagnosis plays a key role in fast restoration response to fault events. The fault diagnosis systems that have been developed are often built upon Artificial Intelligence (AI) techniques [3], [4]. The modelling system developed by Correcher et al [5], [6] proposed a fault diagnosis algorithm for a electricity network.
More recently, the condition monitoring [7]-[10] and control of networks are based on the use of an Energy Management System (EMS) [11] and SCADA system [12], [13]. Thus, many in the research community have focused on fault diagnosis involving automated analysis of EMS/SCADA data to provide decision support to control engineers tasked with fault location, repair and system restoration. Dekkers et al [14] develop a new approach for analysis of faults in electricity supply network by interpreting SCADA data, and McArthur et al [15] have utilised Multi-Agent System (MAS) based SCADA and fault record analysis [16], [17] to provide diagnostic assistance to protection engineers.

While the research community has focused on developing diagnostic techniques involving post-event data analysis as a reactive response to fault events, this paper describes a proactive approach to mitigating fault events and unplanned outages through fault prognostics. This approach utilizes:

- SCADA alarms as a means of identifying repeated auto-recloser operation and thereby problematic circuits.

- Auto-recloser current pick-up data as fault activity monitoring data to determine whether a permanent fault may be evolving on these problematic circuits due to degradation on the line and to identify the root cause.

The distribution network data contains details of anomalous activity, incipient fault activity and permanent fault activity. Accordingly, after preliminary identification of SCADA alarm data, a deep analysis of PMAR data (distribution network data) in this fault diagnosis and prognosis system could assist control engineers with more efficient decision support.

The system's architecture and fault diagnosis methodology are detailed in this paper. Then, a following case study demonstrates the diagnostic functionality of the system. Finally, it presents the approach by utilizing the knowledge based system to support fault diagnosis and prognosis of this integrated system in future. 


\section{Challenge}

The research reported in this paper is a demonstration that the analysis of SCADA data alone is not sufficient to support diagnosis and prognosis of distribution network faults. However, combining SCADA data analysis with analytic algorithms targeted at lower level circuit monitoring data (i.e. current pickup data from PMARs) allows the diagnostic and prognostic system to be created.

PMAR [18] is an example of distribution automation technology with embedded monitoring installed on the overhead lines in the distribution network of ScottishPower Energy Networks (SPEN) [19]. PMARs are generally placed on the overhead lines that are frequently affected by unpredictable disturbances.

Not only does the PMAR have the function of a circuit breaker [9] to protect the customer from significant periods off supply, but it records current measurement data (referred to as "pickup" event information) on which it bases its trip operation. The PMAR and SCADA system are already preinstalled on the network and the diagnostic and prognostic system designed makes use of their circuit monitoring capability, so there is no requirement for dedicated condition monitoring hardware to capture data for analysis.

The records generated by the PMAR contain the open and close sequence, the currents' amplitudes and durations of pickups leading to trip events (e.g. transient fault, semi-permanent fault, etc.), the affected phases, and underlying pick-up activity, which may not be sizeable enough to cause an autorecloser trip operation. A transient fault will disappear in a short time (e.g. a broken branch falls on the overhead line). A semi-permanent fault [2], [20] can arise from degradation of the line and may lead to frequent short-term supply interruptions affecting quality of daily electricity service, this fault could be presented as intermittent outages or 'nuisance tripping' (e.g. resulting from rain affecting a cracked insulator on a wood pole).These semi-permanent faults often result in PMAR tripping but not necessarily any lockout, so service is often resumes without any prolonged outage. As a consequence no further investigation is generally required and so the underlying cause of this tripping activity remains undiagnosed. There then exists a risk of this semi-permanent fault activity evolving over time into a more serious permanent fault resulting in a prolonged network outage, requiring fault location, isolation and repair, as well as emergency customer supply restoration. Figure 1 shows the main steps of the fault diagnosis procedure within the system.

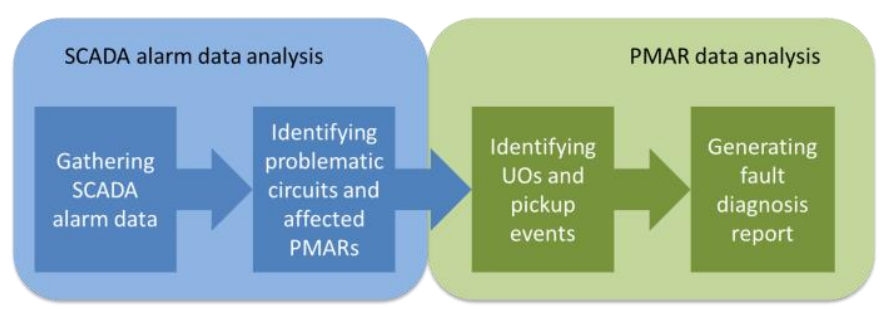

Fig. 1. Fault diagnosis with SCADA alarm data and PMAR data
As a result, when a fault occurs in a circuit, the fault diagnosis system gathers SCADA alarm data around this fault in the particular circuit at first. After identifying faults with related PMARs, the diagnosis will directly focus on analysing the pickup event information to identify the category of fault type (e.g. transient fault, semi-permanent fault, etc.). The unpredictable nature of transient faults and their causes (typically, weather, third party interference) mean that the system focuses only on semi-permanent faults (which are seen as an early stage of evolution of a permanent fault, symptomatic of circuit degradation). If the semi-permanent fault could be identified and diagnosed, this could alert control engineers to prevent a future permanent fault from arising and so the system effectively supports fault prognosis here to mitigate future faults and the resulting outages.

Compared with fault analysis utilizing SCADA only, this proposed approach provides a greater level of diagnostic/prognostic detail and allows more focused analysis before or after the fault event.

\section{FAUlt Diagnosis/Prognosis SYSTEM}

\section{A. Data Sources}

The following data sources, are available from the Distribution Network Operator (DNO), and support the analysis of the PMARs' pickup events [21]:

- PSALERTS is a database that archives:

- SCADA alarms which include Unsolicited Opening (i.e. DNOs record numerous trips on the circuits throughout the year, but since no circuit outage (lockout) occurs, these are not subject to the same detailed investigation as permanent faults which require location and repair. Trips resulting from such activity are referred to as unsolicited openings.)

- Information associated with the particular circuit details (e.g. circuit number, PMARs' names, etc.) and event log time, etc.

- PROSPER is a database that includes causal information relating to repaired permanent faults, i.e. cause of faults, fault clearing time, the relevant PMAR's name, etc. The corresponding information of unsolicited openings and pickup events for the related permanent fault could be found in the PSALERTS and PMAR log file.

- PMAR log file contains pickup events (earth fault pickup, pre transient pickup, transient pickup and lockout) details [21], such as current amplitudes of faults, event log time, the faulted phase/s, the duration between the fault events and pickups and clearance, etc.

\section{B. System Architecture}

This integrated fault diagnosis and prognosis system consists of three analysis functions: 
- Fault diagnosis is achieved by:

- SCADA alarm data analysis for anomaly detection and problematic circuit location

- PMAR data analysis for identification of semipermanent faults

- Fault prognosis is based on:

- Semi-permanent fault activities analysis for fault prognostics

Figure 2 shows the relationship of three parts with their own data sources.

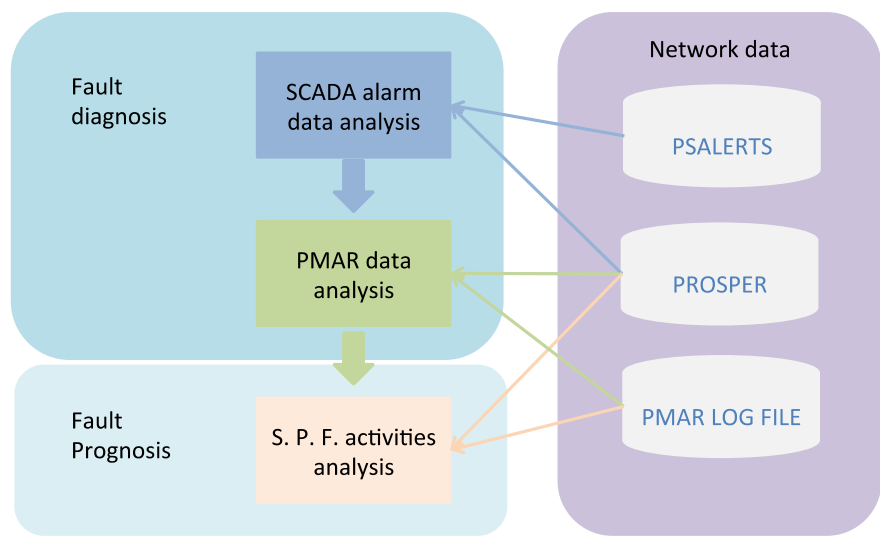

Fig. 2. Fault diagnosis/prognosis system architecture (S. P. F. $=$ SemiPermanent Fault)

\section{1) SCADA Alarm Data Analysis}

The SCADA alarm data analysis is the first half of the fault diagnosis, which is supported by a previously developed Anomaly Detector software tool. It is an automatic filter which extracts all the information related to relevant circuits and PMARs affected by frequent tripping from the SCADA alarm data (stored in the PSALERTS database). This data is then stored in different databases supporting the different functions of the Anomaly Detector.

Additionally, this Anomaly Detector will display extracted and derived information (e.g. circuit number, affected PMAR, frequency of tripping in different periods, etc.) to control engineers when it detects a real-time SCADA alarm associated with PMARs.

2) PMAR Data Analysis Function

As illustrated in the above diagram, the input data of the PMAR Data Analysis Function is based on the result from the SCADA Anomaly Detector. When the specified PMAR is determined, the analysis tool will focus on detecting and analysing the recorded pickup events in the PMAR log files. Combined with relevant PROSPER historical data; the cause of this pickup activity may be identified. That is, the semipermanent fault could be identified [22].

\section{3) Semi-Permanent Fault Analysis Function}

After successful identification of semi-permanent fault, the fault prognosis function will focus on classifying fault signatures to identify the patterns/trends of semi-permanent faults, by applying data mining technologies [23]. These patterns/trends could be utilised to automatically generate rules, to assist quick and efficient identification of pickup events. Finally, a fault diagnosis and prognosis report would be produced to support the decision making of the control engineers.

\section{Fault Diagnosis Methodology}

This paper focuses on the methodology and development of the fault diagnosis. It utilizes the functions of SCADA alarm data analysis and PMAR data analysis. Figure 3 describes the high-level fault diagnosis methodology including the data flow and analysis.

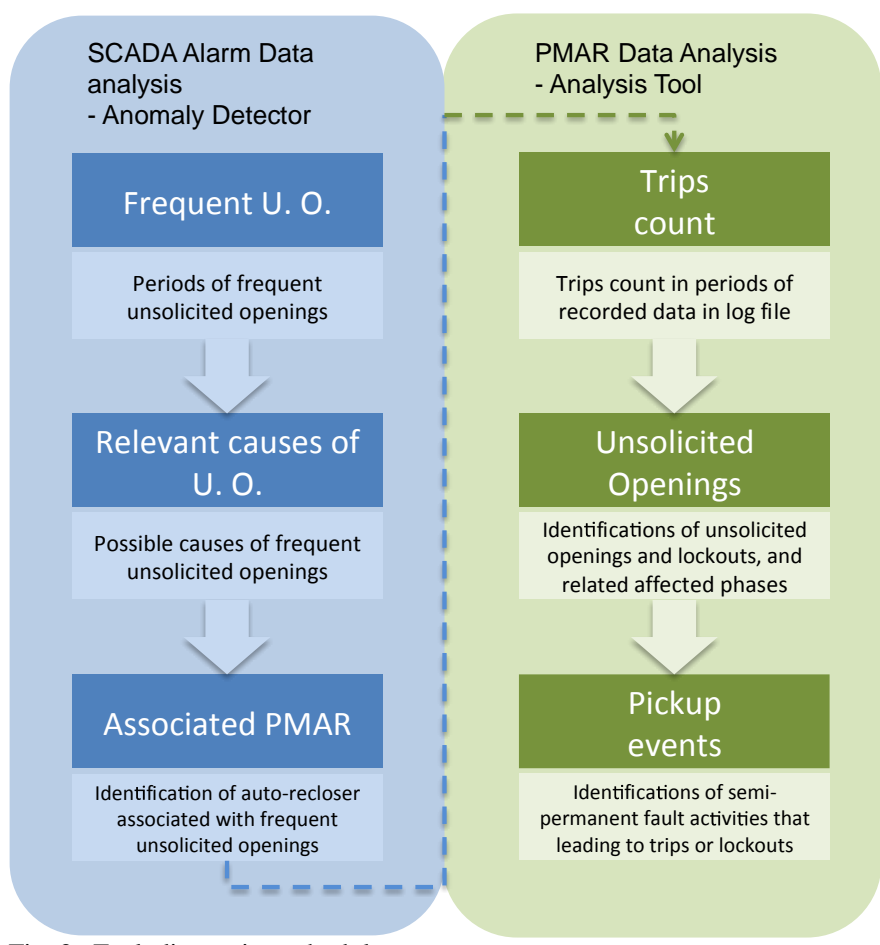

Fig. 3. Fault diagnosis methodology

As displayed in the flow chart, the anomaly detector has three main functions: the frequent unsolicited openings identification function, the relevant unsolicited openings' causes identification function and the associated PMAR identification function. Also, there are three important subfunctions in the PMAR Data Analysis Function: trips count, unsolicited opening identification, pickup event identification. The steps of fault diagnosis in view of the identification and classification of unsolicited openings and pickup events consists of several steps:

- $\quad$ Step 1. Extract unsolicited openings associated with PMARs from the PSALERTS data (SCADA alarms) to determine high frequency of unsolicited openings on circuits inferring existence of a semi-permanent fault.

- Step 2. Identify possible causes of unsolicited openings and associated circuit's PMARs from 
PROSPER data, to support the analysis of PMAR data.

- Step 3. Extract all the tripping events (include frequent unsolicited openings) from recorded data in PMAR $\log$ file to identify affected phases of unsolicited openings and lockouts, and repeated patterns of affected phases (tripping events with the same affected phases recorded frequently in a period) which may indicate an evolving semi-permanent fault.

- Step 4. Extract pre transient pickup events which are associated with identified unsolicited openings and lockouts, and combine with possible causes captured from PROSPER data, to aid in identifying cause of semi-permanent faults.

Since the PROSPER data are taken into consideration for identification of a semi-permanent fault, the type of fault which causes an auto-recloser's lockout should be detected at first. Presently, there are four types of fault recorded in the PROSPER data. They are deterioration due to ageing or wear (excluding corrosion); weather and environment (lightning, wind and gale, snow, sleet and blizzard, birds; third party damage (farm and domestic animals); unknown. Since faults caused by third party and weather and environment can't be predicted, the classification of fault signatures is focused on deterioration and the unknown category. These faults may have associated pre-fault activity in the PMARs which does not lead to a lockout.

\section{CASE STUdY}

To illustrate the fault diagnosis system's functionality, this case study provides an example of operation based on a distribution network operator's data. In order to identify frequent unsolicited openings all of the relevant PSALERTS records from the distribution network circuits have been taken into consideration. As a result, the Anomaly Detector filtered 114 circuits associated with PMAR records. Among these circuits, 9 circuits with a high frequency of unsolicited openings have been identified and focused on for fault diagnosis.

In order to identify a semi-permanent fault in the circuits of interest, the Anomaly Detector searches for relevant fault descriptions (e.g. cracked insulator) in the categories of equipment deterioration from the PROSPER data source. Then, the particular PMARs associated with possible causes of a semi-permanent fault would be identified. Normally, more than one PMAR would be installed in a distribution network circuit. In these 9 circuits, 14 associated PMARs have been identified. Therefore, these identified PMAR log files would contain the information related to frequent unsolicited openings resulting from semi-permanent faults.

Each PMAR log file contains abundant information (lockout events, tripping events, pre transient pickup events, etc.) over several years. In order to identify the pickup events related to a semi-permanent fault, the first priority is to choose the period which has an appropriate concentration of unsolicited openings and lockouts. Figure 4 shows the distribution of unsolicited openings and lockouts with a normalised date range for one particular PMAR.

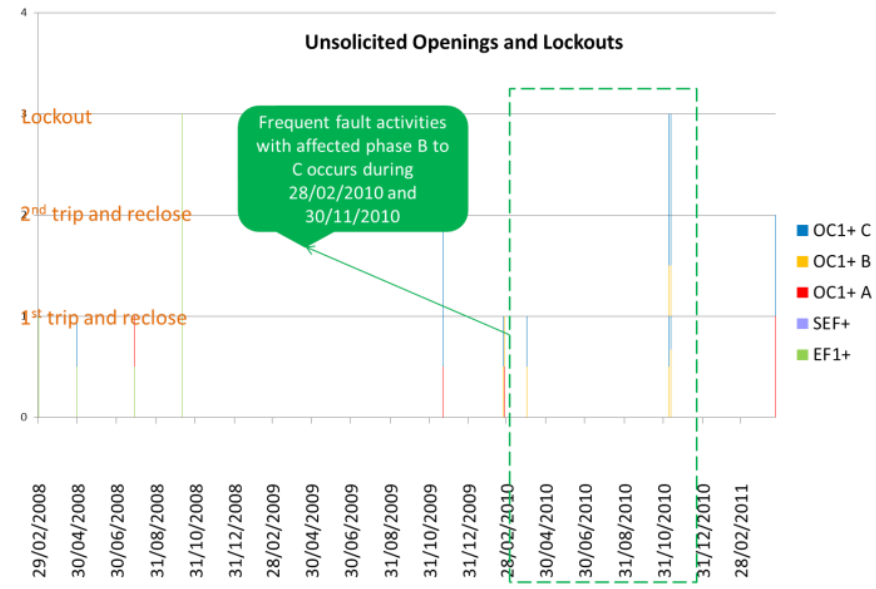

Fig. 4. Unsolicited openings and lockouts of a PMAR

In the example, a number of unsolicited openings, which affected phases $\mathrm{B}$ and $\mathrm{C}$, have been identified by the analysis tool. These trips are distributed into different periods but occur frequently, so these intermittent faults may suggest a semipermanent fault with the same cause that occurs and then clears without intervention. In order to determine the frequent unsolicited openings related to the semi-permanent fault, Figure 5 shows a visualised example of pre transient pickup events and tripping activities extracted and derived from the same PMAR log file.

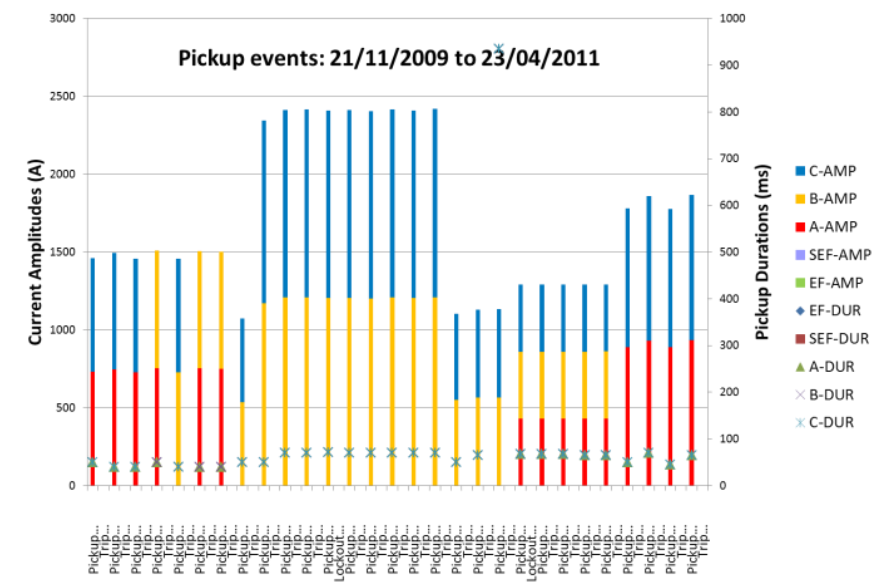

Fig. 5. Relative pickup events of a PMAR

In this example, the three phases are represented by different colours with the standard convention: $\mathrm{A}=\mathrm{Red}, \mathrm{B}=$ Yellow and $\mathrm{C}=\mathrm{Blue}$. If in case of earth faults are recorded at the meantime, the purple and green columns will represent sensitive earth fault and earth fault. The height of each bar shows the current recorded during each pickup event, the scatter crosses represent the duration of pickup event. The lockouts, tripping events, and pre-transient pickup events are 
displayed on the primary horizontal axis. The primary and secondary vertical axes respectively describe amplitudes (A) of current and pickup events' duration (ms).

As illustrated in the above figure, numerous trips related to phase $\mathrm{B}$ and $\mathrm{C}$, or phase $\mathrm{A}$ and $\mathrm{C}$, are recorded from $21 / 11 / 2009$ to $23 / 04 / 2011$. Also two overhead line phase lockouts occurred on the 08/11/2010 and the 11/11/2008. Both of the two lockouts were the result of the unsolicited openings on the affected phases B and C. After manually clearing the faults and reclosing the PMARs for normal electricity supply, the same cause of trip occurred again in 2010 and 2011 Because the fault descriptions recorded in the PROSPER data indicated a cracked insulator, these unsolicited openings with affected phase $\mathrm{B}$ and $\mathrm{C}$ could be identified as a semipermanent fault. Therefore, the methodology and tool allows the identification of semi-permanent fault conditions.

After identifying semi-permanent faults through the approach described in this paper, the fault signatures (tripping phases, current amplitudes, tripping frequencies, etc.) could be identified and classified through data mining processes and techniques. In this case study, the frequent trips on phases B and $\mathrm{C}$, with similar pickup duration, would become a fault feature that could be classified as a pattern of semi-permanent faults in this circuit. Then, using methodologies such as rule induction, the semi-permanent fault analysis function would generate a rule to identify the patterns of the particular semipermanent fault. This rule would assist control engineers to identify the fault and potential future issues quickly and efficiently.

\section{KNOWLEDGE BASED SYSTEM IMPLEMENTATION}

Based on the description of the fault diagnosis methodology and the introduction to the identification of patterns of semi-permanent faults in the case study, this section describes the steps (i.e. high-level tasks process) involved in the design of a knowledge based system and the interactions between them.

The knowledge based system will automatically analyse the unsolicited opening events using the input from the PSALERTS database, and utilises an inference (reasoning) engine to match the observed pre-processed facts with related knowledge, where the represented knowledge is derived from the industry network experts and formalised in the knowledge base. The system will apply the knowledge to online data to identify whether a semi-permanent fault exists or not, and what type of fault it is. It will provide an 'early warning' of an evolving semi-permanent fault. The knowledge based system is being developed using the CommonKADS [24][25][26] approach to knowledge engineering, and Drools Fusion offers a framework to implement the processing, reasoning and rules identified [27]. Figure 6 shows the knowledge based system architecture.

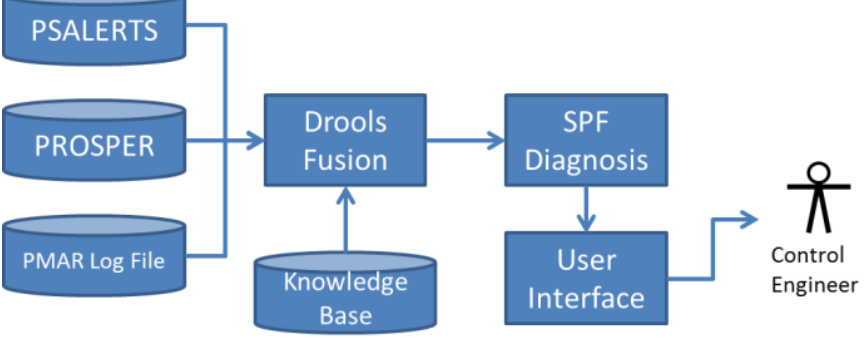

Fig. 6. Architecture of knowledge based system processing

The knowledge engineering techniques are used to construct the general and specific rules. The following example shows the general rules to handle 'Unsolicited Opening' events from SCADA alarms (taken from PSALERTS). These events could be associated with semi-permanent faults, and relevant fault descriptions are recorded in the PROSPER database and can be used to identify this situation.

\section{If Device Type $=P M A R$ \\ And Information Type = 'Unsolicited Opening' \\ Then This PMAR device is affected by 'Unsolicited Opening', need to check with fault records}

Fig. 7. Rule for stage 1

If Device Type $=$ PMAR

And Information Type = 'Unsolicited Opening'

And Incident Description $=$ Cracked Insulator

Then The 'Unsolicited Opening' events of PMAR device could be result from semi-permanent fault, need to check with pickup events

Fig. 8. Rule for stage 2

\section{If Device Type $=$ PMAR}

And Information Type = 'Unsolicited Opening'

And Incident Description = Cracked Insulator

And Recurrent pickup patterns precede the fault event

And Frequency or amplitude of patterns is increasing

Then PMAR Records indicate semi-permanent fault activity. Report this to engineers

Fig. 9. Rule for stage 3

As illustrated in the example, the rules generally describe the working flow of identifying semi-permanent faults. Stage 1 (Figure 7) retrieves 'Unsolicited Opening' events of the PMAR from the PSALERTS database, then in stage 2 (Figure 8) the inference engine checks if the identified events could relate to a potential semi-permanent fault description. In this instance, it determines that if a cracked insulator has been identified then this is known to cause semi-permanent faults. Finally, stage 3 (Figure 9) focuses on identifying the patterns and trends of pickup events preceding the fault events to decide on the notification of evolving semi-permanent faults

\section{FUTURE WORK}

Future work will focus on validating the identification of semi-permanent faults by identifying useful PROSPER data related to frequent unsolicited openings. Once the validation of fault diagnosis is completed, the next step is to develop the 
knowledge based prognosis system which automatically applies data mining techniques to classify and cluster fault signatures of identified patterns/trends of semi-permanent faults. From there, the research will focus on developing an integrated system to provide technical support to achieve the objective of mitigating customer supply interruptions via the distribution automation scheme [28].

\section{CONCLUSION}

This paper offers a novel approach to fault prognosis to identify the fault by automatic analysis of Pole Mounted AutoRecloser data. The case study demonstrated the steps and effectiveness of the fault diagnosis methodology. Additionally, in the case study, the identification of semi-permanent faults has been discussed with relevant PMAR log files associated with specific circuits and time-windows of interest, which will form the basis of further analysis and the data mining effort required to create a prognostic system.

\section{ACKNOWLEDGEMENTS}

The authors would like to acknowledge the funding, support and technical input from ScottishPower Energy Networks.

\section{REFERENCES}

[1] R. B. Duffey, and T, Ha, "The probability and timing of power system restoration," Power Delivery, IEEE transactions, vol. 28, no. 1, pp. 3-9, 2013.

[2] S. Kazemi, M, Lehtonen, and M. Fotuhi-Firuzabad, "Impacts of fault diagnosis schemes on distribution system reliability." Smart Grid, IEEE Transactions, vol. 3, no. 2, pp. 720-727, 2012.

[3] L. Xu, and M. Y. Chow, "A classification approach for power distribution systems fault cause identification," Power Systems, IEEE Transactions, vol. 21, no. 1, pp. 53-60, 2006.

[4] B. Das, "Fuzzy logic-based fault-type identification in unbalanced radial power distribution system," Power Delivery, IEEE Transactions, vol. 21, no. 1, pp. 278-285, 2006.

[5] A. Correcher, E. Garcia, F. Morant, R. Blasco-Gimenez, and E. Quiles, "Diagnosis of intermittent fault dynamics," Emerging Technologies and Factory Automation, ETFA 2008, IEEE International Conference, pp. 559-566, 2008

[6] A. Correcher, E. Garcia, F. Morant, E. Quiles, and L. Rodriguez, "Intermittent failure dynamics characterization," Reliability, IEEE Transactions, vol. 61, no. 3, pp. 649-658, 2012.

[7] J. Seshadrinath, B. Singh, and B. K. Panigrahi, "Incipient Turn Fault Detection and Condition Monitoring of Induction Machine Using Analytical Wavelet Transform." Industry Applications, IEEE Transactions, vol. 50, no. 3, pp 2235-2242, 2014.

[8] S. E. Rudd, J. D. Kirkwood, E. M. Davidson, S. M. Strachan, V. M. Catterson, and S. D. J. McArthur, "Intelligent monitoring of the health and performance of distribution automation." Intelligent System Application to Power Systems (ISAP) 2011, 16th International Conference, pp. 1-6, 2011.

[9] S. M. Strachan, S. D. J. McArthur, B. Stephen, J. R. McDonald and A Campbell, "Providing decision support for the condition-based maintenance of circuit breakers through data mining of trip coil current signatures," Power Delivery, IEEE Transactions., vol. 22, no. 1, pp. 178-186, 2007

[10] G. Ming, Y. Xu and R. Du, "An intelligent online monitoring and diagnostic system for manufacturing automation," Automation Science and Engineering, IEEE Transactions., vol. 5, no. 1, pp. 127-139, 2008.

[11] C. Chen, S. Duan, T. Cai, B. Liu, and G. Hu, "Smart energy management system for optimal microgrid economic operation," IET renewable power generation., vol. 5, no. 3, pp. 258-267, 2011
[12] Z. Liu, J. Liu and Z. Jiao, "Research of power system fault diagnosis method based on information identification," In Electricity Distribution (CICED), 2012 China International Conference on., pp. 1-4., IEEE, 2012.

[13] C. Alcaraz, C. Fernandez-Gago, and J. Lopez, "An early warning system based on reputation for energy control systems," Smart Grid, IEEE Transactions, vol. 2, no. 4, pp. 827-834, 2011

[14] T. Dekkers, M. Zut, and K. Gleeson, "A new approach to intermitten fault management in low voltage networks to reduce the number of repeat interruptions," Electricity Distribution (CIRED 2013), 22nd International Conference, pp. 1-3, 2013.

[15] S. D. J. McArthur, E. M. Davidson, V. M. Catterson, A. L. Dimeas, N. D. Hatziargyriou, F. Ponci, and T. Funabashi, "Multi-agent systems for power engineering applications-Part I: Concepts, approaches, and technical challenges," Power Systems, IEEE Transactions, vol. 22, no. 4, pp. 1743-1752, 2007.

[16] F. M. A. Mohamed, and H. Shareef, "Power quality diagnosis in distribution networks using rule based S-transform method," Environment and Electrical Engineering (EEEIC) 2010 9th International Conference, pp. 361-364, 2010.

[17] R. Moghe, and M. J. Mirrasoul, "Trend analysis techniques for incipient fault prediction," Power \& Energy Society General Meeting, 2009 IEEE PES, pp. 1-8, 2009.

[18] NOJA POWER, 'Recloser', NOJA POWER, URL: http://www.nojapower.co.uk/product/recloser.html, April. 2014.

[19] Our Distribution Network, SP Energy Network, UPL: http://www.spenergynetworks.co.uk/pages/our_distribution_network.as p, Feb, 2015.

[20] C. Ly, K. Tom, C. S. Byington, R. Patrick and G. J. Vachtsevanos, "Fault diagnosis and failure prognosis for engineering systems: A global perspective." Automation Science and Engineering CASE 2009. IEEE International Conference, pp. 108-115. IEEE, 2009.

[21] X. Wang, S. M. Strachan, J. D. Kirkwood, and S. D. J. McArthur, "Automatic analysis of Pole Mounted Auto-Recloser data for fault prognosis to mitigate customer supply interruptions," Power Engineering Conference, UPEC, 49th International Universities, pp. 1-6, 2014

[22] H. MacDonald, "NOJA - TELUS Import," Scottish Power Energy Networks, 2010

[23] D. L. Olson, and D. Delen, "Advanced Data Mining Techniques", Springer, chapter 1, 2008, pp.3-8

[24] G. Schreiber, "Knowledge Engineering and Manegement: The CommonKADS Methodology”, MIT Press, 1999, pp. 27-28, 295-314.

[25] C. J. Edwards, E. M. Davidson, S. D. J. McArthur, I. Watt, and T. Cumming. "Flexible Model-Based Alarm Processing for Protection Performance Assessment and Incident Identification." Power Systems, IEEE Transactions, vol. 28, no. 3, pp. 2584-2591.

[26] L. Xiang, G. Sun, and X. Zhong, "A rule-based exception reason diagnosis system for electric power data." Electricity Distribution (CICED), 2012 China International Conference, pp. 1-4, 2012.

[27] M. Bali, "Drools JBoss Rules 5.0 Developer's Guide", Packt Publishing Limited, chapter 1, 2009, pp. 7-15.

[28] Z. Liu, D. Niu, X. Yang, and W. Sheng, "Research on intelligent decision support system for power system." Information and Automation, 2009. ICIA09. International Conference, pp. 412-417. IEEE, 2009. 